\title{
Controlled Porosity of MCM-41 Obtained by Partial Blocking of Pores by Silicon Oil
}

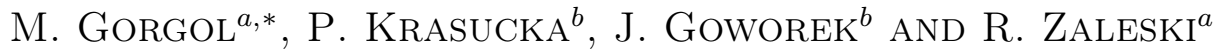 \\ ${ }^{a}$ Maria Curie-Skłodowska University, Institute of Physics, Department of Nuclear Methods, \\ pl. M. Curie-Skłodowskiej 1, 20-031 Lublin, Poland \\ ${ }^{b}$ Maria Curie-Skłodowska University, Faculty of Chemistry, Department of Adsorption, \\ pl. M. Curie-Skłodowskiej 3, 20-031 Lublin, Poland
}

\begin{abstract}
Partial blocking of mesopores in the ordered MCM-41 silica by DC550 silicon oil was proposed as a preparation method of the material with controlled porosity and fixed pore size. The porosity of the samples with various content of DC550 was examined with the use of low temperature nitrogen sorption and positron annihilation lifetime spectroscopy. It was shown that the oil blocks the primary pores by forming the plugs near its entrances, but also partially locates in the interparticle spaces. The comparison of the results obtained from both investigation techniques was used to make the first attempt to obtain the calibration of ortho-positronium intensity, depending on pore volume. This is necessary to improve the utility of positron annihilation lifetime spectroscopy as a porosimetric technique. The need to take the migration of positronium to larger free volume into account is discussed.
\end{abstract}

DOI: 10.12693/APhysPolA.132.1559

PACS/topics: 78.70.Bj, 36.10.Dr, 61.43.Gt, 68.43.--h

\section{Introduction}

The extended Tao-Eldrup (ETE) model [1] describes the relation between the lifetime of ortho-positronium $(o-$ Ps) and the pore size. It is applicable in the approximate range of $0.2-50 \mathrm{~nm}$. This model is widely used for characterization of porous materials by means of positron annihilation spectroscopy for antihydrogen production [2], low- $k$ materials [3], hybrid materials [4, 5], studies of adsorption phenomena [6,7], etc. Development of the ETE model progresses in various ways, e.g. by taking into account the heterogeneity of pore sizes [8]. Another direction is a search for a comprehensive relation between PALS parameters (i.e. the lifetime and intensity of pore related $o$-Ps components) and a pore size distribution [9] to obtain universal porosimetric method [10].

The main inconvenience of this method is lack of the absolute calibration of the $o$-Ps intensity $\left(I_{o-P s}\right)$. To achieve this, a series of model materials with precisely controlled porosity is required. This is not easy to prepare series of the same material differentiated in respect to precise pore dimensions control. The popular model material is MCM-41, which pores are relatively long, unconnected cylinders of uniform size [11]. Numerous PALS studies of this material [12-15] make it sufficiently well known to be a preferable choice for calibration of positron porosimetry. However, tailoring of the total pore volume and specific surface of MCM- 41 in the wide range, which is required for the calibration, is impossible at the synthesis stage. Hydrothermal treatment of as synthesized

\footnotetext{
* corresponding author; e-mail: marek.gorgol@poczta.umcs. lublin.pl
}

sample cause a serious reconversion of pore system with partial loss of structural regularity.

In the present paper we propose to control the porosity of MCM-41 by partial blocking of pores. This can be done using the silicon oil, which easily penetrates pores due its affinity to silanol groups. Once introduced into pores, the oil cannot be removed by pumping at room temperature due to its large molecular mass. This allows to carry out PALS measurements in high vacuum, which is required to apply the ETE model.

\section{Experimental}

\subsection{Materials}

Tetraethoxysilane (TEOS) and hexadecyltrimethylammonium bromide (CTAB) were purchased from Sigma Aldrich. Ammonium hydroxide solution (25\%) and acetone $(99.5 \%)$ were obtained from $\mathrm{POCH}$ (Poland). Polyphenylmethyldimethylsiloxane Silicone DC550 fluid (115 cSt) was purchased from Serva. All materials were used without further purification.

MCM-41 silica material was synthesized using the standard procedure described in Ref. [16]. Molar composition of reaction components was as fol-

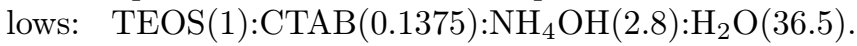
As synthesized silica-surfactant composite was filtered, washed with distilled water and dried in air at $90^{\circ} \mathrm{C}$. Finally the dry material was calcined twice, first at $550^{\circ} \mathrm{C}$ $\left(1^{\circ} \mathrm{C} / \mathrm{min}\right)$ for $8 \mathrm{~h}$ and again at $550^{\circ} \mathrm{C}$ for $4 \mathrm{~h}$ in oxygen atmosphere to eliminate carbon traces. MCM- 41 silica material was divided into 4 parts: one was left as received, the others were soaking with acetone/silicone DC550 oil mixture with different concentration of DC550 oil $(5 \%, 10 \%, 20 \% \mathrm{v} / \mathrm{v} \%)$. The expression $\mathrm{v} / \mathrm{v} \%$ means volume percent, calculated from the equation 


$$
v / v \%=\frac{V_{\mathrm{DC} 550}}{V_{\mathrm{DC} 550}+V_{\text {acetone }}} \times 100 \%,
$$

where $V_{\text {DC550 }}$ and $V_{\text {acetone }}$ is the volume of DC550 and acetone, respectively.

Wet samples were left for conditioning for $5 \mathrm{~h}$ at room temperature, next all samples were dried at $60^{\circ} \mathrm{C}$ for $5 \mathrm{~h}$ to evaporate the acetone. MCM- 41 samples reached $m_{\mathrm{DC} 550}=23 \%, 41 \%$ and $58 \%(\mathrm{w} / \mathrm{w} \%)$ DC 550 oil content, respectively, where

$$
w / w \%=\frac{W_{\mathrm{DC} 550}}{W_{\mathrm{DC} 550}+W_{\mathrm{MCM}-41}} \times 100 \% .
$$

The $W_{\mathrm{DC} 550}$ and $W_{\mathrm{MCM}-41}$ is the weight of DC550 and MCM-41, respectively.

\subsection{Methods}

Nitrogen adsorption/desorption measurements were carried out with a volumetric adsorption analyzer ASAP 2405 (Micromeritics, Norcros, USA). The specific surface area, $S_{\mathrm{BET}}$, was calculated from the BET equation [17] in the relative pressure range from 0.05 to 0.25 . The pore size distributions (PSDs) were obtained using the Barrett-Joyner-Halenda (BJH) procedure [18], whereas the total pore volume, $V_{p}$, was estimated from a single adsorption point at the relative pressure of 0.985 .

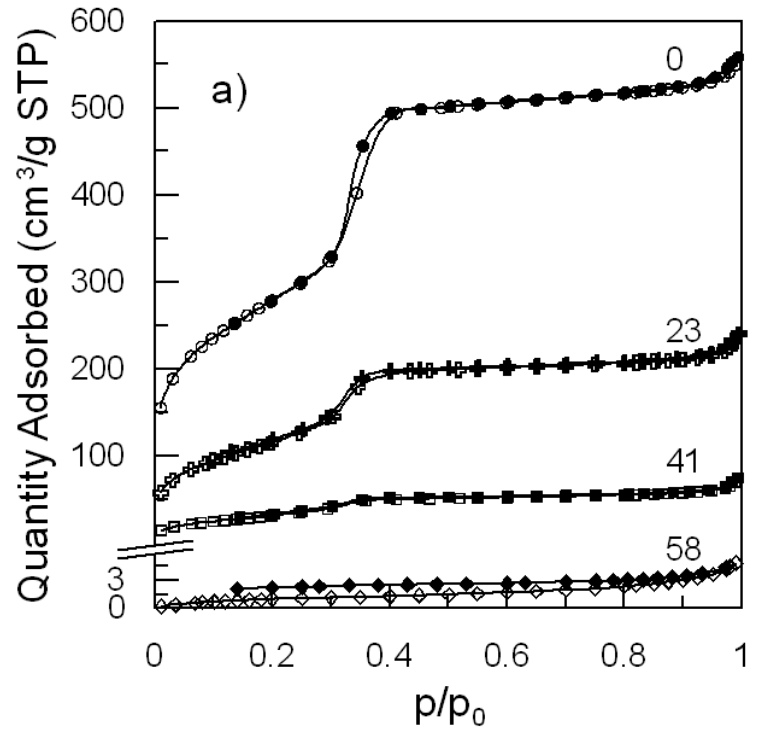

Fig. 1. Nitrogen adsorption/desorption isotherms at $77 \mathrm{~K}$ (a) and pore size distributions calculated using the BJH method (b) for MCM-41 with various content of DC550 oil. Adsorption - empty symbols, desorption - full symbols.

The isotherms for $m_{\mathrm{DC} 550}=0,23 \%$, and $41 \%$ have similar shapes, but they become flatter with the increasing content of DC550. This indicates that the increasing part of their pores is blocked by the silicon oil. Pore filling is represented quantitatively by the specific pore surface $\left(S_{\mathrm{BET}}\right)$ and total pore volume $\left(V_{p}\right)$, which both decrease with the increasing amount of DC550 (Table I). In contrary to expectation for ideal pore filling, this decrease is not a linear function of the volume of the oil (Fig. 2). This suggests that either a part of pores becomes inaccessible to nitrogen or the structure DC550 is

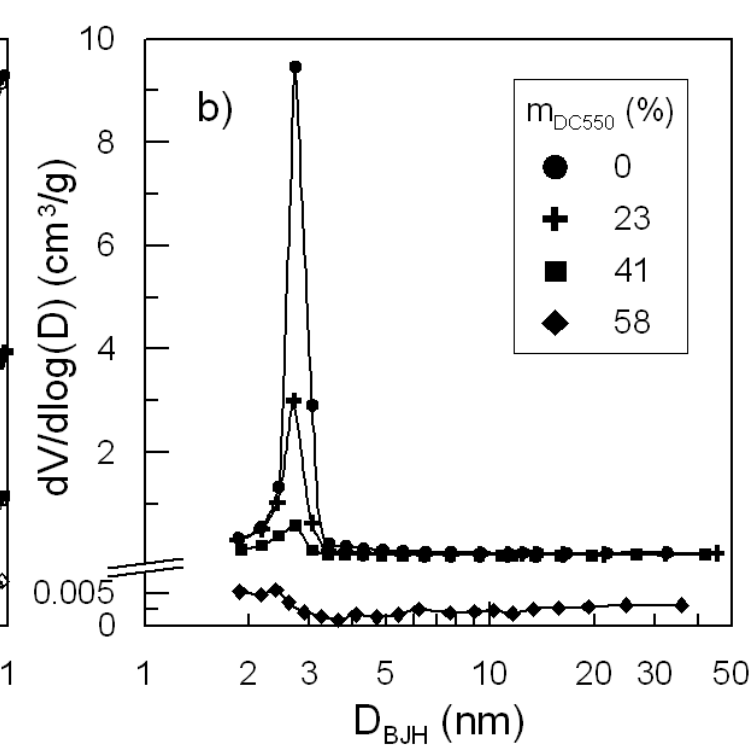

A fast-slow delayed coincidence spectrometer with two $\mathrm{BaF}_{2}$ scintillation detectors was used to perform PALS measurements. The $2 \mu$ s time range of time-to-amplitude converter allowed to analyze large free volumes, especially mesopores. FWHM of the resolution curve was about 290 ps $(+5 \%$ of $440 \mathrm{ps})$ with the ${ }^{22} \mathrm{Na}$ source of $0.2 \mathrm{MBq}$ used. Each sample was kept in vacuum $\left(p<10^{-4} \mathrm{~Pa}\right)$, obtained with the use of turbomolecular pump. The total count number for each sample was about $1.7 \times 10^{7}$. The spectra were analyzed with the use of the PALSfit3.

\section{Results and discussion}

The porosity of the unmodified MCM-41 and its three derivatives with varying content of DC550 silicon oil was determined from a low temperature nitrogen adsorption isotherms (Fig. 1a). The isotherm of MCM-41 is of type IV with a step at $p / p_{0}=0.3-0.4$, which represent nitrogen condensation in mesopores. A steep increase and absence of hysteresis in this pressure range testifies about the uniform size of pores and lack of any significant constrictions. This is reflected by a single narrow peak in the pore size distribution (PSD) obtained from the BJH method (Fig. 1b). looser at partial fillings than for fully filled pores. Finally, all pores are blocked if $m_{\mathrm{DC} 550}=58 \%$ as indicated by the nearly flat nitrogen isotherm and the negligibly small values of $S_{\mathrm{BET}}$ and $V_{p}$. At higher oil loadings the difference $V_{p \text { (init) }}-V_{\mathrm{DC} 550}$ becomes negative, which means that the volume of the oil phase exceeds the initial pore volume of unmodified MCM-41. This undoubtedly indicates that large part of the added silicone oil is located outside the pores in the interparticle space.

The position of the peak in PSD remains unchanged for various $m_{\text {DC550 }}$ (Fig. 1b, Table I). This testifies that 
TABLE I

Specific pore surface $\left(S_{\mathrm{BET}}\right)$, total pore volume $\left(V_{p}\right)$ and pore size at maximum of PSD $\left(D_{\mathrm{BJH}}\right)$ for the samples under study.

\begin{tabular}{c|c|c|c}
\hline \hline$m_{\mathrm{DC} 550}[\%]$ & $S_{\mathrm{BET}}\left[\mathrm{m}^{2} / \mathrm{g}\right]$ & $V_{p}\left[\mathrm{~cm}^{3} / \mathrm{g}\right]$ & $D_{\mathrm{BJH}}[\mathrm{nm}]$ \\
\hline 0 & 1008 & 0.86 & 2.7 \\
23 & 433 & 0.37 & 2.7 \\
41 & 119 & 0.12 & 2.7 \\
58 & 4 & 0.01 & n.a.
\end{tabular}

DC550 forms plugs in the pores, i.e. it completely fills whole clearance of pore at certain length instead of making it narrower. This is very favorable for the calibration purpose, because the obtained series of materials has all but one (i.e. the pore concentration) properties nearly the same.

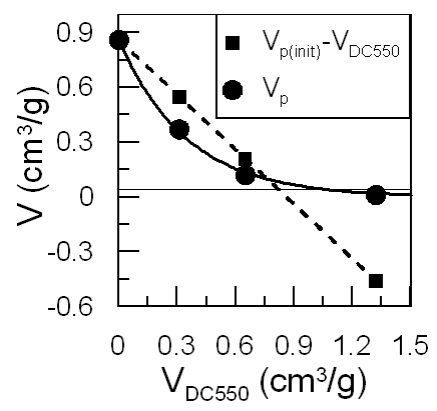

Fig. 2. The total pore volume derived from nitrogen adsorption experiment $\left(V_{p}\right)$ and the expected empty pore volume obtained as difference between the initial total pore volume $\left(V_{p \text { (init) }}\right)$ and the oil phase volume $\left(V_{\mathrm{DC} 550}\right)$ as a function of the oil phase volume for samples with various pore filling. The solid line represent an exponential function fitted to the data, the dashed line shows the linear dependence, which is expected if the oil is located only in primary mesopores without their blocking.

Six components are necessary to obtain a good fit $\left(\chi^{2}=1.02-1.06\right)$ for the PAL spectra of all studied samples. Two short-lived components originates from annihilation of para-positronium $\left(\tau_{1} \approx 117(7) \mathrm{ps}\right)$ and free positrons $\left(\tau_{2} \approx 370-400 \mathrm{ps}\right)$. Slightly different from others are lifetimes in the unmodified MCM-41, where two components that can be ascribed to free positrons were resolved $\left(\tau_{2}=238(12)\right.$ ps and $\left.\tau_{3}=524(5) \mathrm{ps}\right)$. The remaining components represent annihilation of $o$ Ps in free volumes of various size. In the pure MCM-41 they are subsequently: micropores $\left(\tau_{4}=5.3(3) \mathrm{ns}\right)$, primary mesopores $\left(\tau_{5}=38(1) \mathrm{ns}\right)$ and interparticle spaces $\left(\tau_{6}=125.5(3) \mathrm{ns}\right)$ (Fig. 3).

The interparticle component predominates in the spectrum which is the result of $o$-Ps migration outside mesopores $[15,19]$. The introduction of oil causes that $o$-Ps forms also at the oil-silicon interface $\left(\tau_{3}=1.50(6) \mathrm{ns}\right)$ and in bubbles distended by $o$-Ps in the oil $\left(\tau_{4}=\right.$ $3.22(2) \mathrm{ns}$ ), while the contribution of micropores becomes

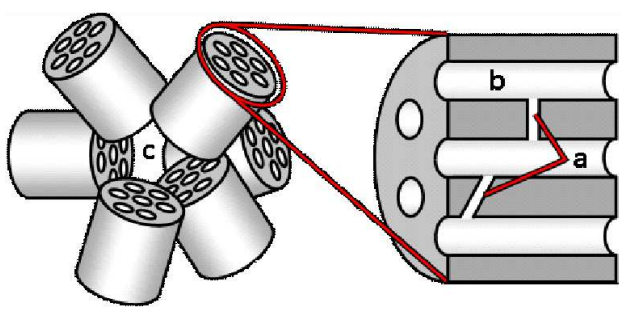

Fig. 3. A scheme of MCM-41 pore system, taking into account micropores (a), primary pores (b), and interparticular spaces (c).

negligible. The intensities of these components $\left(I_{3}\right.$ and $I_{4}$ ) reflect increasing amount of DC550 (Fig. 4). The intensity of the bubble related component $\left(I_{4}\right)$, which is connected to the bulk oil, increases linearly with $m_{\mathrm{DC} 550}$. Slightly different, $I_{3}$ seems to reach a saturation above $m_{\text {DC550 }}=41 \%$. This indicates that increase of the volume of the bulk oil phase is not accompanied by the further expansion of the oil-silica interface. This confirms that DC550 is located not only in primary mesopores but its significant part fills much larger interparticle spaces at $m_{\mathrm{DC} 550}=58 \%$.

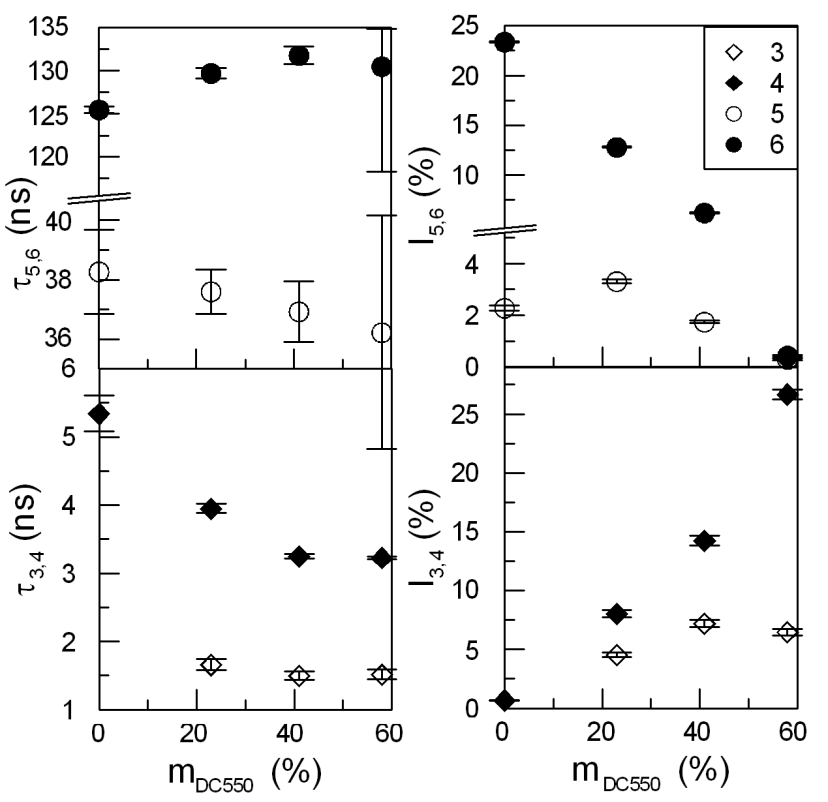

Fig. 4. The lifetimes $\left(\tau_{i}\right)$ and intensities $\left(I_{i}\right)$ of the $o$ Ps components $(i=3-6)$ as a function of the mass of DC550 introduced to MCM-41.

The increasing contribution from the oil related components should entail the decrease of $I_{5}$ and $I_{6}$ as the empty space in pores is filled by DC550. Indeed, $I_{6}$ follows the expected tendency, but $I_{5}$ shows it only for $m_{\text {DC550 }}=23-58 \%$. Opposite change (i.e. increase of $I_{5}$ ) is observed when $23 \%$ of DC550 is introduced to MCM41. Most likely this is a consequence of the partial suppression of the $o$-Ps migration when primary mesopores 


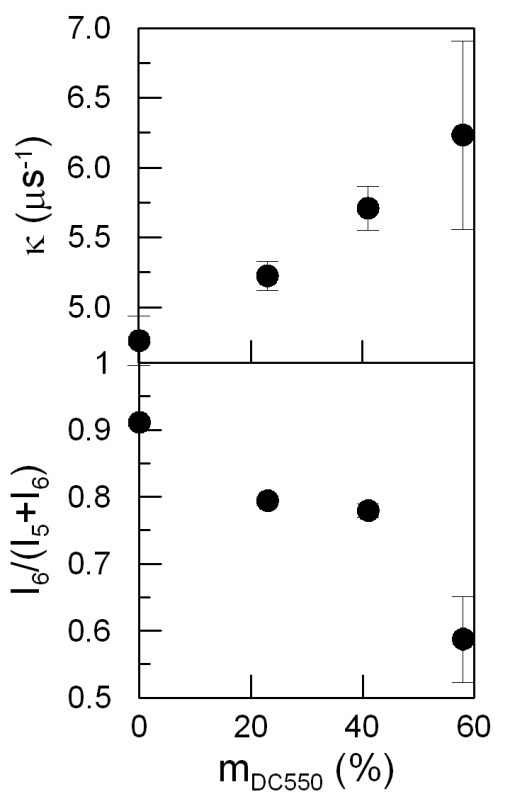

Fig. 5. The rate of $o$-Ps migration from the primary mesopores to interparticle spaces $(\kappa)$ and the intensity ratio of $o-\mathrm{Ps}$ in interparticle spaces to the total $o$-Ps in pores $\left(I_{6} /\left(I_{5}+I_{6}\right)\right)$ as a function of the mass of DC550 introduced to MCM-41.

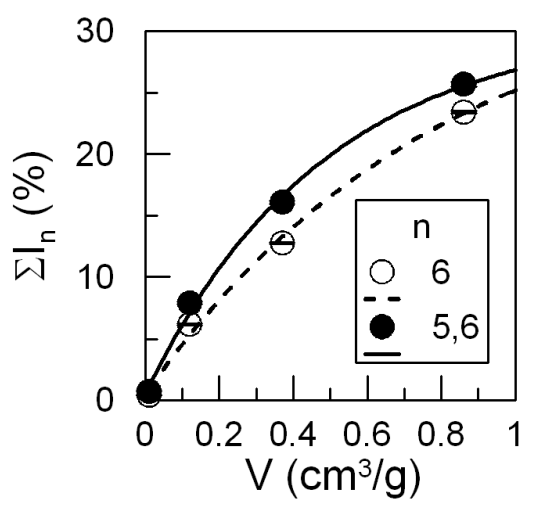

Fig. 6. The relation between intensity $\left(I_{n}\right)$ of the components originating from pores of MCM- 41 and the total pore volume from nitrogen adsorption.

are blocked by the oil. Appearance of pores closed between two plugs formed by DC on the ends of a pore can be expected, but this is not necessary to obtain the decreased migration rate. Also a single plug at the end of a pore is sufficient to suppress the migration, while the pore remains open from the other side.

The rate of migration can be estimated from equation

$$
\kappa=\frac{1}{\tau_{5}}-\frac{1}{46.8 \mathrm{~ns}}
$$

under the assumption that only migration is responsible for deviation of $\tau_{5}$ from the lifetime of $46.8 \mathrm{~ns}$, which is expected by the ETE model for a pore of size $2.7 \mathrm{~nm}$. The rate calculated this way (Fig. 5) increases almost linearly with $m_{\mathrm{DC} 550}$. This is against the previously described tendency of $I_{5}$, which suggests that $\kappa$ should initially decrease. The disagreement is even better pronounced when $\kappa$ is compared to the mass dependence of $I_{6} /\left(I_{5}+I_{6}\right)$, i.e. the intensity ratio of $o$-Ps in interparticle spaces to the total $o$-Ps in pores. This ratio reflects approximately the fraction of $o$-Ps that escaped from primary pores, under assumption that $o$-Ps formation in interparticle spaces is negligible. This is justified, because the intensity related to interparticle spaces is six times smaller when primary pores are filled by template in raw MCM-41 [20]. Thus, it should be even lower when there is a high competition from forming $o-\mathrm{Ps}$ in primary pores. The $I_{6} /\left(I_{5}+I_{6}\right)$ ratio clearly shows the reduction of the escaped $o$-Ps fraction when DC550 is introduced and then its approximately constant probability until almost no o-Ps annihilates in pores. On this basis, the assumption that the reduction of $\tau_{5}$ is caused mostly due to the $o$-Ps migration should be rejected. Instead, the reduction of $\tau_{5}$ is caused by the contribution from the closed pores, where $o$-Ps lifetime is smaller due to their length limited by the oil plugs. The contribution of the closed primary pores can be estimated as $10-15 \%$ from the $I_{6} /\left(I_{5}+I_{6}\right)$ ratio.

It follows from the foregoing considerations that both the $o$-Ps migration effect and closed porosity have to be taken into account to obtain the reliable calibration of $I_{o-P s}$. Even though the nitrogen adsorption shows that the dominant contribution to pore volume comes from primary pores, the $o$-Ps migration makes that $I_{6}$ should be taken into account in the calibration. In turn, the closed porosity, which is not accessible by adsorption based methods, should not be taken into account. Therefore, using $I_{5}+I_{6}$ in the calibration can lead to slightly overestimated intensity. The resultant curve, which binds $I_{o-P s}$ and $V$ for pore size of $2.7 \mathrm{~nm}$, lies in between of both lines presented in Fig. 6. Nevertheless, its estimation is precise enough to conclude that the relation between the $o$-Ps intensity and the total pore volume has an exponential shape, which leads to saturation between 30 and $35 \%$. It has to be remembered that the calibration is valid for the current setting of the spectrometer and should depend on the width of the stop energy window.

\section{Conclusions}

The decrease of porosity parameters obtained from $\mathrm{N}_{2}$ sorption results (i.e. specific pore surface and total pore volume) with the increasing content of DC550 indicates that the silicon oil is a good wetting agent for MCM-41 silica. It mostly blocks the primary pores most likely by locating near their entrances. No significant decrease of pore diameter was observed. The part of the silicon oil locates also in the interparticle spaces, at least when primary pores are nearly completely filled.

The high intensity of the longest-lived component, observed in positron annihilation lifetime spectroscopy analysis for unmodified MCM-41, shows the predominant 
character of $o$-Ps migration from mesopores to interparticle spaces. However, the calculations of the ratio of $o-$ Ps migration and fraction of $o$-Ps escaping from primary pores to larger free volumes do not correspond each other. It can be explained if the reduction of $\tau_{5}$ is only partially caused by the $o$-Ps migration when DC550 partially fills primary pores. The major contribution to this reduction is expected to come from the closed pores, whose length is relatively small.

The calibration curve linking the pore volume obtained from $\mathrm{N}_{2}$ sorption and the $o$-Ps intensity (corresponding mainly to annihilation in the primary pores) can be approximated by an exponent, which reaches saturation at about $30 \%$. This value could be slightly different for various settings of positron annihilation lifetime spectrometers. If only closed pores contribute to the component related to the primary pores, the pore volume given by $\mathrm{N}_{2}$ sorption correlate only with the intensity of the component related to interparticle spaces. In contrary, if all primary pores are open, the volume corresponds to the intensities of both components related to interparticle spaces and primary pores. Therefore, two curves were presented in the proposed calibration. The area between them relates to the most probable situation, i.e. part of pores is closed, but part remains open. The calibration is valid for $2 \mathrm{~nm}$ cylindrical mesopores. Further development of the model in this direction should consist in finding similar curves for pores of different size and shape.

\section{References}

[1] T. Goworek, K. Ciesielski, B. Jasinska, J. Wawryszczuk, Chem. Phys. 230, 305 (1998).

[2] G. Consolati, R. Ferragut, A. Galarneau, F. Di Renzo, F. Quasso, Chem. Soc. Rev. 42, 3821 (2013).

[3] M. Lépinay, N. Djourelov, H. Marinov, L. Broussous, K. Courouble, C. Licitra, F. Bertin, V. Rouessac, A. Ayral, J. Por. Mater. 21, 475 (2014).
[4] P. Krasucka, R. Zaleski, K. Skrzypiec, J. Goworek, Micropor. Mesopor. Mater. 237, 210 (2017).

[5] C. He, B. Xiong, W. Mao, Y. Kobayashi, T. Oka, N. Oshima, R. Suzuki, Chem. Phys. Lett. 590, 97 (2013).

[6] R. Zaleski, W. Dolecki, A. Kierys, J. Goworek, Micropor. Mesopor. Mater. 154, 142 (2012).

[7] R. Zaleski, A. Kierys, M. Gorgol, Phys. Chem. Chem. Phys. 19, 10009 (2017).

[8] M. Khaghani, A.A. Mehmandoost-Khajeh-Dad, Nucl. Instrum. Methods Phys. Res. B 396, 11 (2017).

[9] R. Zaleski, W. Stefaniak, M. Maciejewska, J. Goworek, J. Por. Mater. 16, 691 (2009).

[10] R. Zaleski, Nukleonika 60, 795 (2015).

[11] C.T. Kresge, M.E. Leonowicz, W.J. Roth, J.C. Vartuli, J.S. Beck, Nature 359, 710 (1992).

[12] Y.J. He, H.Y. Zhang, Y.B. Chen, H.Y. Wang, T. Horiuchi, J. Phys. Condens. Matter 13, 2467 (2001).

[13] J. Wawryszczuk, J. Goworek, R. Zaleski, T. Goworek, Langmuir 19, 2599 (2003).

[14] R. Zaleski, M. Sokół, Mater. Sci. Forum 666, 123 (2011).

[15] R. Zaleski, A. Błażewicz, A. Kierys, Nukleonika 58, 233 (2013).

[16] M. Grün, K.K. Unger, A. Matsumoto, K. Tsutsumi, Micropor. Mesopor. Mater. 27, 207 (1999).

[17] S. Brunauer, P.H. Emmett, E. Teller, J. Am. Chem. Soc. 60, 309 (1938).

[18] E.P. Barrett, L.G. Joyner, P.P. Halenda, J. Am. Chem. Soc. 73, 373 (1951).

[19] J. Goworek, A. Borowka, R. Zaleski, R. Kusak, J. Therm. Anal. Calorim. 79, 555 (2005).

[20] R. Zaleski, J. Wawryszczuk, A. Borowka, J. Goworek, T. Goworek, Micropor. Mesopor. Mater. 62, 47 (2003). 